\title{
Management Dilemma of Concurrent Fundal Submucous Fibroid and Incomplete Uterine Septum in a Patient with Recurrent Pregnancy Loss
}

\author{
Hina Javaid, MD, ${ }^{1}$ Mohammad Ashraf, MD, ${ }^{2}$ and Mostafa I. Abuzeid, MD, FACOG, FRCOG ${ }^{2-4}$
}

\begin{abstract}
Background: We describe a patient presenting with recurrent pregnancy loss secondary to concurrent uterine factors. Case: The patient was a 31-year-old white female who presented with recurrent pregnancy loss on two occasions. The couple had a normal work-up for recurrent miscarriages except for a fundal type II submucosal fibroid on transvaginal 3D ultrasound (US) with saline infusion hysterosonogram (SIH). Laparoscopic myomectomy and repair of the myometrial defect was performed. At the conclusion of the procedure, a diagnostic hysteroscopy suggested a possible concurrent incomplete short uterine septum, which could not be corrected for fear of cutting the myomectomy sutures. The patient did not appear for follow up, which led her to have three more miscarriages and one chemical pregnancy. The presence of a short uterine septum was confirmed on SIH with 3D US. The patient underwent hysteroscopic division of the short uterine septum. Results: Postoperative SIH showed a normal uterine cavity, and currently the patient is trying to conceive. Conclusion: Coexistence of a type II submucous fibroid in the fundal region of the uterus and an incomplete uterine septum presents both a diagnostic and management challenge. Surgical management of such coexisting uterine pathology cannot be performed in a single session. (J GYNECOL SURG 29:165)
\end{abstract}

\section{Introduction}

$\mathbf{R}$ ECURRENT PREGNANCY LOSS (RPL) is defined as two or more consecutive spontaneous miscarriages and occurs in $0.5 \%-3 \%$ of women. Twelve to fifteen percent of women with recurrent pregnancy loss have a uterine malformation. A uterine septum as well as submucosal and subserosal intramural leiomyomas distorting the uterine cavity are frequent causes of recurrent miscarriages. ${ }^{1-3}$ The aim of this report is to bring to attention the unique diagnostic and surgical dilemma of dealing with coexisting fundal submucous uterine leiomyomas and an incomplete uterine septum in a patient with RPL, and to discuss the need for spacing out surgeries for such concurrent uterine factors.

\section{Case}

A 30-year-old white female presented with the chief complaint of recurrent miscarriages (on two occasions) at 10 weeks' gestation over a period of 2 years. She and her partner underwent complete work-up for recurrent pregnancy loss, including karyotype analysis on both husband and wife, complete thrombophilia testing, anticardiolipin antibodies, lupus anticoagulant antibody test, and TSH level. All laboratory tests were normal. On transvaginal ultrasound scan (US) and a saline infusion hysterosonogram (SIH) with both $2 \mathrm{D}$ and 3D US, the patient was found to have a $3 \times 2 \mathrm{~cm}$ submucosal, intramural, subserosal fundal fibroid (Fig. 1). According to the European Society for Gynecological Endoscopy (ESGE), the fibroid in this patient was classified as a type II submucosal fibroid. ${ }^{4}$ The fibroid was removed via laparoscopic myomectomy, and the myometrial defect was repaired in two layers using a continuous running 2-0 Vicryl suture (Figs. 2-4). The endometrial cavity was not entered. Hysteroscopy was performed at the conclusion of the procedure, which showed a possible concurrent incomplete short uterine septum with a wide base, rather flat apex, and mid-fundal length of about $1.5 \mathrm{~cm}$ versus a possible fundal indentation secondary to suturing of the myometrial defect status post myomectomy (Fig. 5). The other possibility was that the fundal filling defect was due to the distortion caused by suturing the myometrial defect. It was deemed appropriate that no further action should be taken at that time,

\footnotetext{
${ }^{1}$ Department of Obstetrics and Gynecology, Hurley Medical Center, Flint, MI.

${ }^{2}$ IVF Michigan, PC, Rochester Hills, MI.

${ }^{3}$ Division of Reproductive Endocrinology and Infertility, Department of Obstetrics and Gynecology, Hurley Medical Center, Flint, MI.

${ }^{4}$ Department of Obstetrics and Gynecology and Reproductive Biology, Michigan State University, College of Human Medicine, Flint, MI.
} 


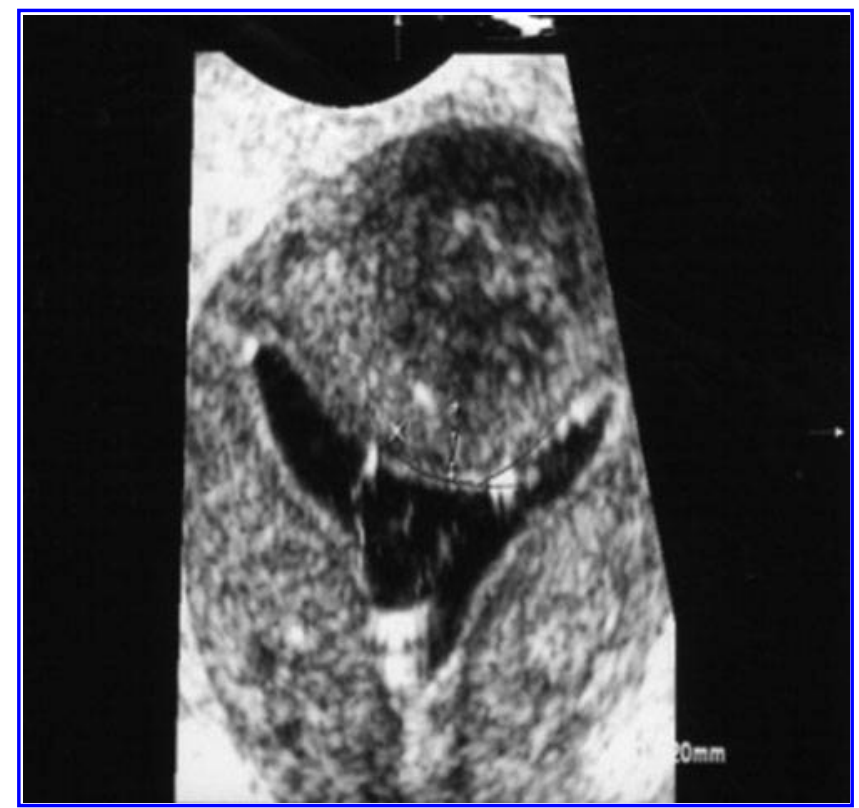

FIG. 1. Transvaginal 3D ultrasound with saline infusion hysterosonogram showing a $3 \times 2 \mathrm{~cm}$ submucosal intramural subserosal fundal fibroid (type II submucosal fibroid). Taken from ISBN: HB 9781107029743. Edited by Botros R.M.B. Rizk and Elizabeth Puscheck. Ultrasonography in Gynecology. Figure 1 in the book chapter "Transvaginal ultrasound scan findings of uterine fibroid effect on treatment plan." By Dr. Mostafa Abuzeid and Salem Joseph. Copyright (C) 2012 Cambridge University Press. Reprinted with the permission of Cambridge University Press.

since we were not sure of the diagnosis. In addition, even if an associated incomplete short uterine septum was present, it could not be corrected for fear of cutting the myomectomy sutures, risk of uterine perforation, and excessive bleeding. The patient was advised to return for a follow-up saline

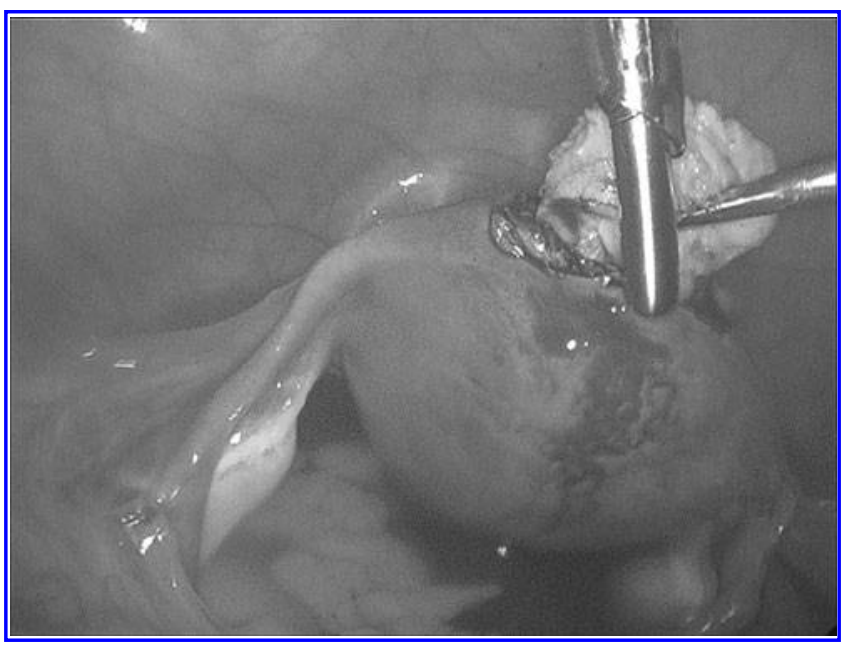

FIG. 2. Laparoscopic picture showing dissection of a fundal uterine fibroid. Taken from ISBN: HB 9781107029743. Edited by Botros R.M.B. Rizk and Elizabeth Puscheck. Ultrasonography in Gynecology. Figure 2 in the book chapter "Transvaginal ultrasound scan findings of uterine fibroid effect on treatment plan." By Dr. Mostafa Abuzeid and Salem Joseph. Copyright ( 2012 Cambridge University Press. Reprinted with the permission of Cambridge University Press.

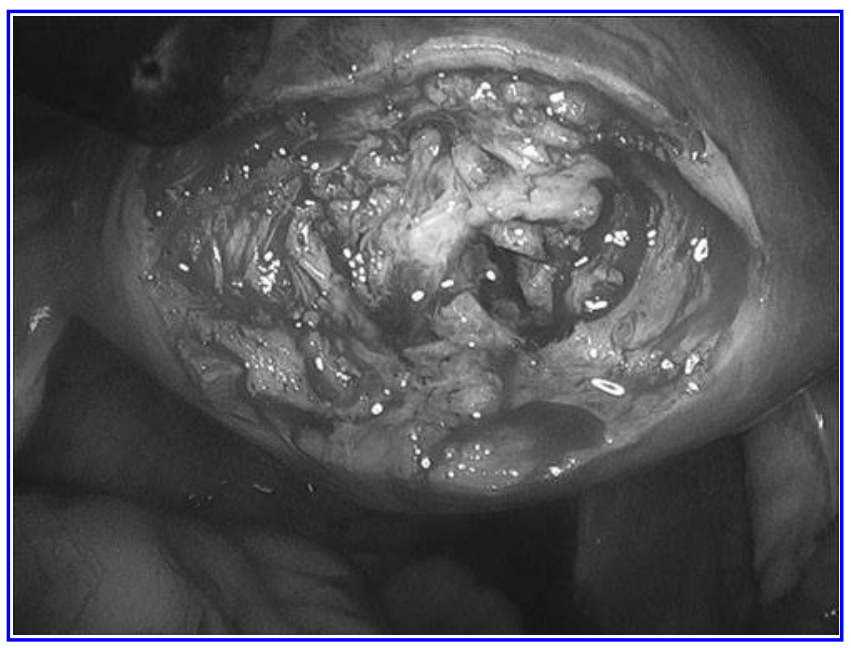

FIG. 3. Laparoscopic picture showing the myometrial defect after removal of a fundal uterine fibroid. Taken from ISBN: HB 9781107029743. Edited by Botros R.M.B. Rizk and Elizabeth Puscheck. Ultrasonography in Gynecology. Figure 3 in the book chapter "Transvaginal ultrasound scan findings of uterine fibroid effect on treatment plan." By Dr. Mostafa Abuzeid and Salem Joseph. Copyright (C 2012 Cambridge University Press. Reprinted with the permission of Cambridge University Press.

sonogram after healing from surgery to confirm the diagnosis of a uterine septum. The patient failed to attend for follow up, which led her to have three more miscarriages and one chemical pregnancy. This happened over a period of 18 months after removal of the fibroid. When the patient returned for follow up, an SIH was performed, which confirmed our earlier suspicion of an incomplete short uterine septum. A diagnostic hysteroscopy confirmed the diagnosis (Fig. 6a and b), and the patient underwent hysteroscopic division of the short uterine septum (Fig. 7). Dilatation and

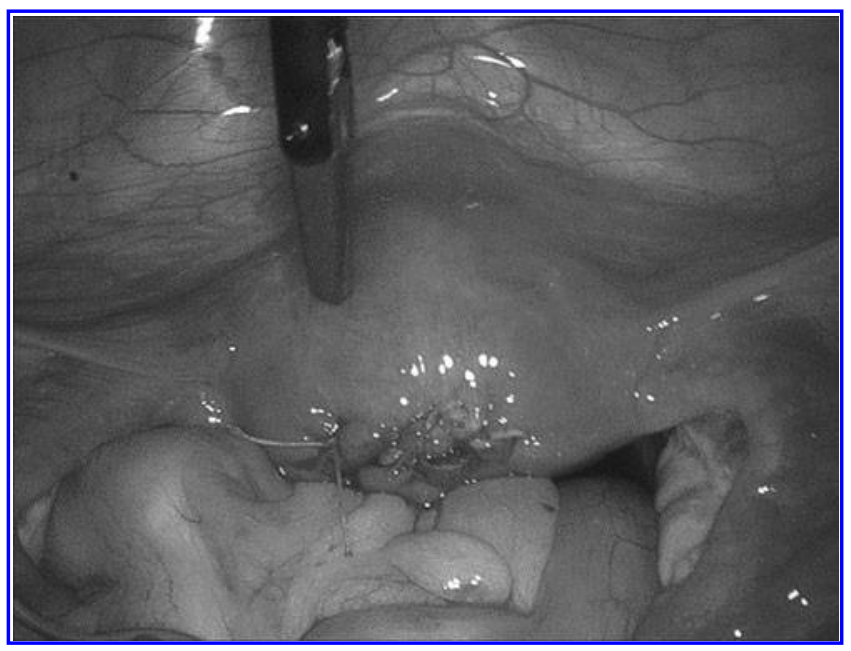

FIG. 4. Laparoscopic picture showing the myometrial defect sutured in two layers. Taken from ISBN: HB 9781107029743. Edited by Botros R.M.B. Rizk and Elizabeth Puscheck. Ultrasonography in Gynecology. Figure 4 in the book chapter "Transvaginal ultrasound scan findings of uterine fibroid effect on treatment plan." By Dr. Mostafa Abuzeid and Salem Joseph. Copyright ( 2012 Cambridge University Press. Reprinted with the permission of Cambridge University Press. 


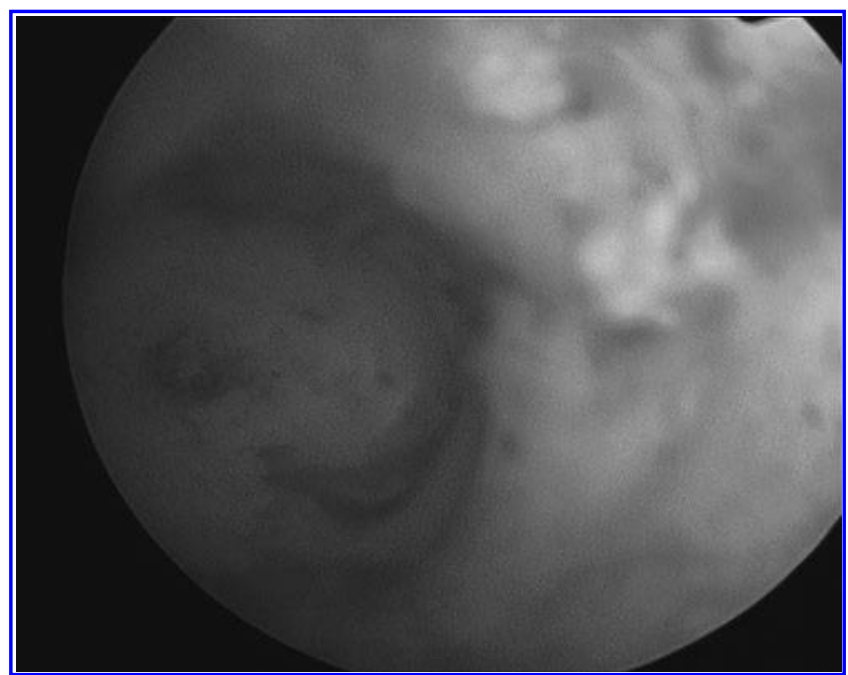

FIG. 5. Hysteroscopic picture at the conclusion of the laparoscopic myomectomy showing the right cornual region with tubal ostium and right portion of a possible incomplete short uterine septum. Taken from ISBN: HB 9781107029743. Edited by Botros R. M. B. Rizk and Elizabeth Puscheck. Ultrasonography in Gynecology. Figure 5 in the book chapter "Transvaginal ultrasound scan findings of uterine fibroid effect on treatment plan." By Dr. Mostafa Abuzeid and Salem Joseph. Copyright (C) 2012 Cambridge University Press. Reprinted with the permission of Cambridge University Press.

curettage was performed at the end of this procedure, and chronic endometritis was ruled out based on the pathology report of the endometrial curetting. Six weeks postoperatively, SIH showed a normal uterine cavity. The patient and her husband were treated with a course of doxycycline (Vibramycin) for 10 days starting with menses for treatment of any possible cervical infection that can lead to miscarriage such as mycoplasma genitalium. Premenstrual endometrial biopsy (EMB) was not performed in view of reports that questioned the accuracy of EMB in the diagnosis of luteal phase defect (LPD). ${ }^{5}$ However, progesterone supplementation was planned 3 days after ovulation or with a positive pregnancy test for treatment of any possible LPD.

\section{Results}

The patient is currently trying to conceive.

\section{Discussion}

Removal of submucosal and intramural fibroids distorting the endometrial cavity has shown to improve fertility outcome. ${ }^{6}$ Submucous fibroids are classified into three categories according to ESGE classification: type 0 if they pedunculate $100 \%$ into the uterine cavity; type I if they arise from the myometrium, but more than $50 \%$ of their mass is within the cavity; and type II if they arise from the myometrium, but less than $50 \%$ of their mass is within the cavity. ${ }^{4}$ Lasmare et al. introduced a new classification of submucous fibroid. In this classification, four criteria were considered: the penetration of the nodule into the myometrium, the extension of the base of the nodule with respect to the wall of the uterus, the size of the nodule, and the topography of the nodule. ${ }^{7}$ More recently, Lasmare et al.

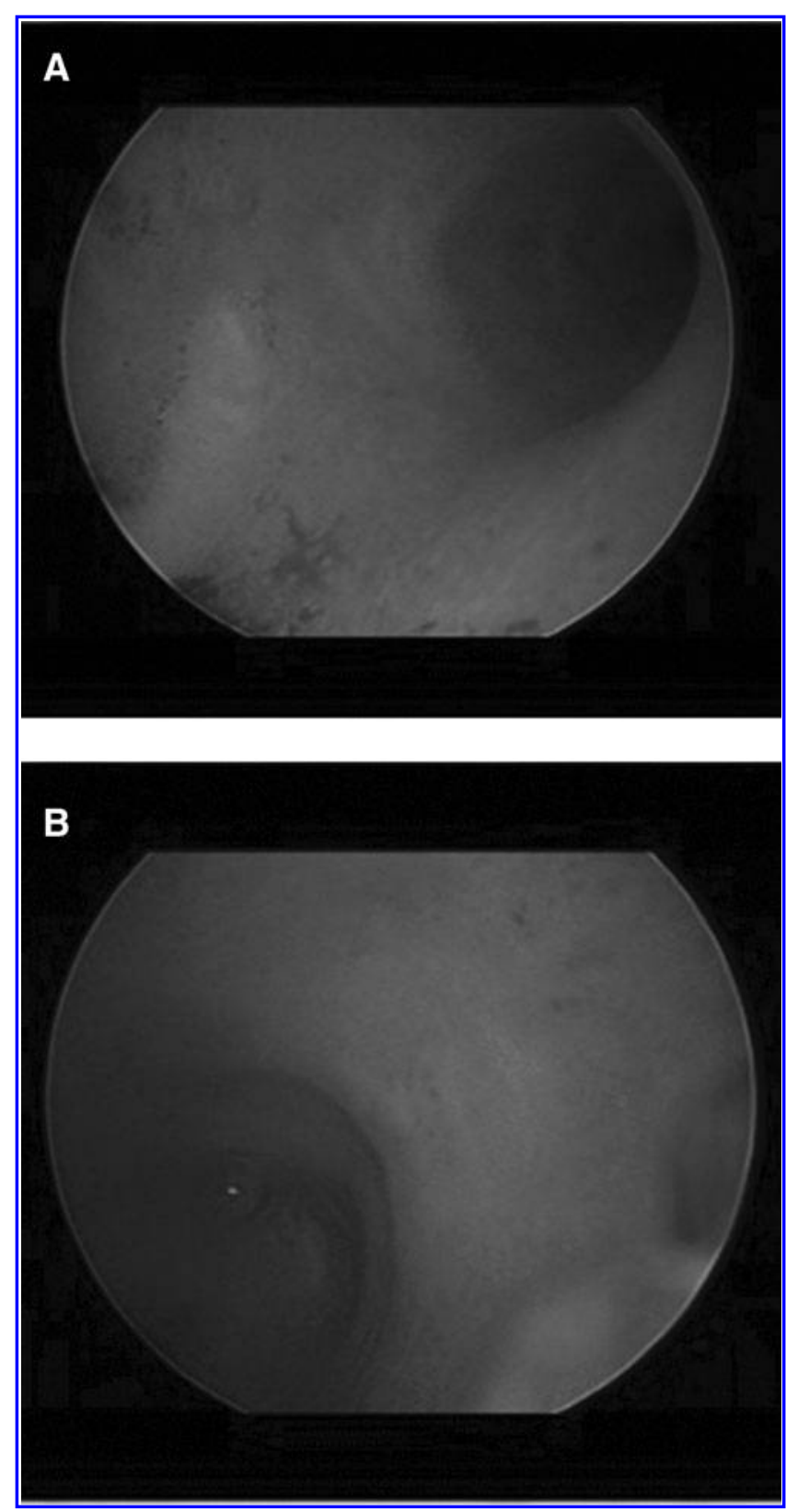

FIG. 6. (A) Hysteroscopic picture 18 months after laparoscopic myomectomy showing the left cornual region with tubal ostium and left portion of an incomplete short uterine septum. (B) Hysteroscopic picture 18 months after laparoscopic myomectomy showing the right cornual region with tubal ostium and right portion of the incomplete short uterine septum.

renamed the new classification STEPW. ${ }^{8}$ In a multicentric study, they correlated ESGE and STEPW classifications with complete and incomplete hysteroscopic removal of a submucosal fibroid and concluded that STEPW permits a greater correlation. ${ }^{8}$ With a score of 7 (STEPW classification), complete hysteroscopic removal was not possible. ${ }^{7,8}$

Hysteroscopic division of a uterine septum has been shown to improve the reproductive outcome in patients with RPL. ${ }^{1,9,10}$ Correction of a subseptate (septum $\geq 1.5 \mathrm{~cm}$ ) or arcuate uterus (septum $\leq 1.5 \mathrm{~cm}$ ) in women with prior 


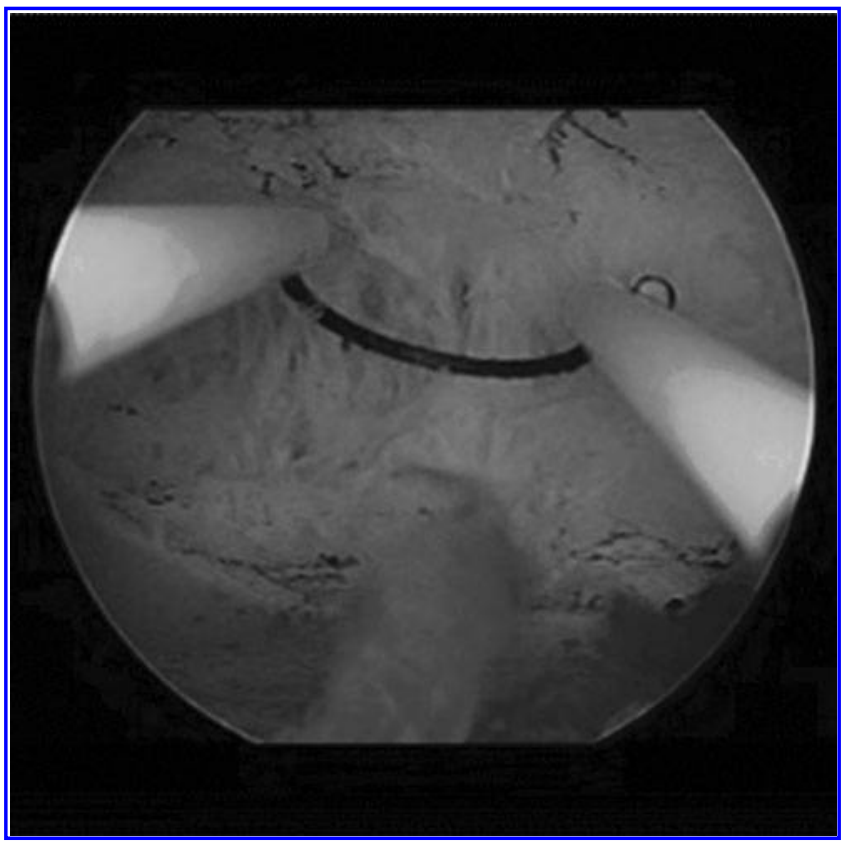

FIG. 7. Hysteroscopic picture 18 months after laparoscopic myomectomy showing the fundal region after dividing an incomplete short uterine septum $(1.5 \mathrm{~cm}$ in length at the midfundal region).

pregnancy loss equally results in a significant decrease in rates of abortion. ${ }^{11,12}$

The real challenge in this case was the suspicion of a concurrent uterine pathology based on the hysteroscopic findings at the conclusion of the laparoscopic myomectomy. In fact, most practitioners will not perform a hysteroscopy, especially when the uterine cavity has not been entered. Practitioners should maintain a high index of suspicion whenever no cause for RPL is found. A review of the literature revealed no previous report on diagnosis or surgical management of a type II submucous fibroid coexisting with an incomplete uterine septum. This is therefore the first report of such an association. We believe that the ideal approach to this challenge is first to attend to the uterine leiomyoma via myomectomy and subsequently to perform hysteroscopic septal division after at least 3-4 months to allow for myometrial healing.

\section{Conclusions}

The coexistence of a type II submucous fibroid in the fundal region of the uterus and an incomplete uterine septum is indeed not only a diagnostic but also a surgical challenge. Removal of just the fibroid can still result in RPL. Surgical management of such coexisting uterine pathology cannot be performed in a single session.

\section{Acknowledgments}

The authors would like to thank Cheryl Anderson for preparation of this article. This abstract was presented at the Global Congress of Minimally Invasive Gynecology 40th
Annual Meeting of the American Association of Gynecologic Laparoscopists (AAGL). J Min Invas Gynecol 2011;186:S154.

\section{Disclosure Statement}

No competing financial interests exist.

\section{References}

1. Daly DC, Maier D, Soto-Albers C. Hysteroscopic metroplasty: six years' experience. Obstet Gynecol 1989;73:201.

2. Eldar-Geva T, Meagher S, Healy DL, MacLachlan V, Breheny S, Wood C. Effect of intramural, subserosal, and submucosal uterine fibroids on the outcome of assisted reproductive technology treatment. Fertil Steril 1998;70: 687.

3. Stovall DW, Parrish SB, Van Voorhis BJ, Hahn SJ, Sparks AE, Syrop $\mathrm{CH}$. Uterine leiomyomas reduce the efficacy of assisted reproduction cycles: results of a matched follow-up study. Hum Reprod 1998;13:192.

4. Wamsteker K, Emanuel MH, deKruif JH. Transcervical hysteroscopic resection of submucous fibroids for abnormal uterine bleeding: results regarding the degree of intramural extension. Obstet Gynecol 1993;82:736.

5. Coutifaris C, Myers ER, Guzick DS, et al. Histological dating of timed endometrial biopsy tissue is not related to fertility status. Fertil Steril 2004;82:1295.

6. Fernandez H, Sefrioui O, Virelizier C, Gervaise A, Gomel V, Frydman R. Hysteroscopic resection of submucosal myomas in patients with infertility. Hum Reprod 2001;16:1489.

7. Lasmar R, Barrozo P, Dias R, Oliveira MA. Submucous fibroids: a new presurgical classification to evaluate the viability of hysteronscopic surgical treatment-preliminary report. J Min Invas Gynecol 2005;12;308.

8. Lasmar RB, Xinmei Z, Indman PD, Celeste RK, Di Spiezio Sardo A. Feasibility of a new system of classification of submucous myomas: a multicenter study. Fertil Steril 2011; 95:2073.

9. Grimbizis G, Camus M, Clasen K, Tournaye H, De Munck L, Devroey P. Hysteroscopic septum resection in patients with recurrent abortions or infertility. Hum Reprod 1998; 13:1188

10. Fedele L, Arcaini L, Parazzini F, Vercellini P, Di Notla G. Reproductive prognosis after hysteroscopic metroplasty in 102 women: life-table analysis. Fertil Steril 1993;59:768.

11. Giacomucci E, Bellavia E, Sandri F, Farina A, Scagliarini G. Term delivery rate after hysteroscopic metroplasty in patients with recurrent spontaneous abortion and T-shaped, arcuate and septate uterus. Gynecol Obstet Invest 2011; 71:183.

12. Gergolet M, Campo R, Verdenik I, Suster NK, Gordts S, Gianaroli L. No clinical relevance of the height of fundal indentation in subseptate or arcuate uterus: a prospective study. Reprod Biomed Online 2012;24:576.

Address correspondence to: Mostafa I. Abuzeid, MD, FACOG, FRCOG Hurley Medical Center Two Hurley Plaza, Suite 209 Flint, MI 48503

E-mail: reprod1@hurleymc.com 